\title{
Quantification of Regional Pulmonary Blood Flow Using ASL-FAIRER
}

\author{
D.S. Bolar, ${ }^{1}$ D.L. Levin, ${ }^{1 *}$ S.R. Hopkins,${ }^{1,2}$ L.F. Frank, ${ }^{1}$ T.T. Liu, ${ }^{1}$ E.C. Wong, ${ }^{1}$ and \\ R.B. Buxton ${ }^{1}$
}

\begin{abstract}
Pulsed arterial spin labeling (ASL) techniques have been theoretically and experimentally validated for cerebral blood flow (CBF) quantification. In this study ASL-FAIRER was used to measure regional pulmonary blood flow (rPBF) in seven healthy subjects. Two general ASL strategies were investigated: 1) a single-subtraction approach using one tag-control pair acquisition at an inversion time (TI) matched to the RR-interval, and 2) a multiple-subtraction approach using tag-control pairs acquired at various Tls. The mean rPBF averaged $1.70 \pm 0.38$ $\mathrm{ml} / \mathrm{min} / \mathrm{ml}$ when measured with the multiple-subtraction approach, and was approximately $2 \%$ less when measured with the single-subtraction method $(1.66 \pm 0.24 \mathrm{ml} / \mathrm{min} / \mathrm{ml})$. Assuming an average lung density of $0.33 \mathrm{~g} / \mathrm{ml}$, this translates into a regional perfusion of approximately $5.5 \mathrm{ml} / \mathrm{g} / \mathrm{min}$, which is comparable to other measures of pulmonary perfusion. As with other ASL applications, a key problem with quantitative interpretation of the results is the physical gap between the tagging region and imaged slice. Because of the high pulsatility of PBF, ASL acquisition and data analysis differ significantly between the lung and the brain. The advantages and drawbacks of the single- vs. multiple-subtraction approaches are considered within a theoretical framework tailored to PBF. Magn Reson Med 55:1308-1317, 2006. (C) 2006 Wiley-Liss, Inc.
\end{abstract}

Key words: arterial spin labeling; pulmonary imaging; pulmonary blood flow; MRI; flow quantification

Matching of pulmonary blood flow (PBF) to ventilation is essential for efficient gas exchange. The ability to quantitatively evaluate changes in regional PBF (rPBF) has the potential to enhance our understanding of the relationship between lung structure and function in both health and disease. Although several techniques for quantitatively evaluating regional pulmonary perfusion are available, most suffer from significant disadvantages, such as the use of ionizing radiation, limited spatial or temporal resolution, or the need for an exogenous contrast agent.

Arterial spin labeling (ASL) magnetic resonance imaging (MRI) techniques have been used to evaluate perfusion in many organ systems. ASL is superior to other current methods of calculating regional blood flow in many aspects; for example, it offers high spatial and temporal

${ }^{1}$ Department of Radiology, University of California-San Diego, San Diego, California, USA.

${ }^{2}$ Department of Medicine, University of California-San Diego, San Diego, California, USA.

D.S. Bolar is now at the A.A. Martinos Center for Biomedical Imaging, Massachusetts General Hospital, Charlestown, Massachusetts, USA.

${ }^{*}$ Correspondence to: David L. Levin, M.D., Ph.D., Department of Radiology, UCSD Medical Center, Mail Code 8756, 200 West Arbor Drive, San Diego, CA 92103-8756. E-mail: dlevin@ucsd.edu

Received 2 August 2005; revised 12 January 2006; accepted 7 February 2006.

DOI 10.1002/mrm.20891

Published online 5 May 2006 in Wiley InterScience (www.interscience.wiley. com)

(C) 2006 Wiley-Liss, Inc. 1308 resolution, is completely noninvasive, and can be repeated over short intervals in the same subject. The ASL technique measures the rate of blood delivery to a local imaging voxel by creating a magnetically "tagged" bolus using specialized radiofrequency $(\mathrm{RF})$ pulses to invert the magnetization of water protons in arterial blood. These inverted protons act as freely diffusible endogenous tracers that can be used to quantify flow. ASL has been widely applied to measure cerebral blood flow (CBF) (1-6), and to a lesser extent has been used to evaluate perfusion in skeletal muscle (7), heart (8), kidney (9), and lung (10-13).

There are a number of variations of ASL. In this paper we focus on a technique called flow-sensitive alternating inversion recovery with an extra RF pulse (FAIRER), which was recently used for pulmonary perfusion imaging $(10,11)$. During each FAIRER measurement a control and tag image are obtained. The control image is acquired by applying a selective inversion pulse to the slice of interest and waiting for a specific inversion time (TI), during which time relaxed blood enters the slice. Imaging is then performed. The tag image is acquired by applying a nonselective inversion pulse to the entire chest volume and waiting for the same TI prior to imaging. The two images are then subtracted. Stationary inverted spins from tissue cancel out, and the resultant image is a perfusion-weighted map. Since FAIRER involves inverted spin delivery from voxels immediately adjacent to the imaging slice, the effective transit times for tagged blood are short, and errors caused by signal decay are minimized. A closely related technique, called the $T_{1}$-method, has also been used to measure pulmonary perfusion (14). Methodologically, this technique is similar to the ASL techniques considered here. It differs primarily in how the experiment is conceptualized-as the delivery of a bolus of tagged spins, or as an apparent alteration of the longitudinal relaxation time $T_{1}$.

In this paper we report our theoretical and experimental analysis of the FAIRER technique applied to the measurement of PBF. Because many previous attempts to optimize ASL methods and interpret ASL data were focused on measuring $\mathrm{CBF}$, it is important to reevaluate these methods in the context of pulmonary perfusion imaging, while taking into account the unique physiologic and hemodynamic properties of the lung. Two specific properties of the lung necessitate significant changes in the way the ASL experiment is performed and analyzed: 1) the highly pulsatile nature of $\mathrm{PBF}$, and 2) the high degree of motion due to subject respiration. By taking advantage of these properties, we used ASL-FAIRER to evaluate a novel single-TI approach that quantifies blood delivered during one cardiac cycle with a single tag/control pair acquisition. We then utilized a more traditional multiple-TI approach to 
quantification, to serve as a comparison. Although a multiple-TI approach requires the acquisition of multiple tag/ control image pairs, and therefore a many-fold increase in total scan time, it allows the generation of the entire magnetic bolus delivery curve. In the brain, such an approach offers an important theoretical advantage for calculating blood flow, and improves accuracy. Interestingly, though, we conclude that such inflow curves require a significantly different interpretation when measured in the lung with a cardiac-gated experiment. By evaluating both techniques, we were able to carefully investigate how the lung's unique properties influence PBF measurement with ASL.

\section{MATERIALS AND METHODS}

\section{Technical Considerations}

An ASL-FAIRER pulse sequence with a half-Fourier acquisition single-shot turbo spin-echo (HASTE) imaging scheme was used for data collection, as described by Mai and Berr (10) and Mai et al. (11). Turbo spin-echo imaging sequences are well suited for the lung, as they reduce susceptibility effects caused by inhomogeneous magnetic fields (15), maximize the intrinsically low lung signal by allowing recovery of $T_{2}$ signal loss $(16,17)$, and reduce artifacts caused by respiratory and cardiac motion (13). Half-Fourier acquisition adds an additional twofold decrease in imaging time, allowing subsecond image acquisition (18).

In FAIRER, the first image is acquired by applying a spatially selective inversion pulse, followed by a saturation pulse, followed by dephasing gradients. An adequate delay (TI) allows relaxed spins to enter from adjacent tissue and displace saturated spins. Imaging begins after this delay. The imaging slice and inversion slice are centered on the same plane such that they overlap. To ensure complete inversion of the spins in the image plane, the width of the selective inversion pulse must be significantly wider than the image slice thickness. In the present study the inversion pulse was three times wider than the imaging slice thickness. The experiment is then repeated with the spatially selective inversion pulse replaced by a nonspatially-selective inversion pulse. This inverts all of the spins in the volume covered by the RF body coil. The same delay (TI) allows inverted spins to enter the imaging plane. Subtraction of the spatially-selective tagged image from the non-spatially-selective tagged image yields a perfusion-weighted image map. The addition of the extra RF pulse and dephasing gradients prevents the zeroing of signal intensity in certain voxels upon subtraction (10). Because the blood-to-tissue ratio is high in any given voxel of the lung, these extra pulses are required in order for the technique to be quantitative (19).

Several issues must be addressed when ASL perfusion imaging is applied to the lung. In contrast to imaging of other organs, breath-holding is required in order to prevent lung motion. As a result, the total imaging time per scan must be short enough so that patients with heart and lung disease are able to comfortably suspend respiration. Breath-holds of 8-10 s are generally short enough for most patients but long enough to provide image maps with adequate SNR. Furthemore, because of the pulsatile nature of pulmonary flow, selectively and nonselectively tagged images must be acquired at the same point in diastole in successive cardiac cycles. If imaging commences during systole, the image will have motion artifacts due to high flow during the several-hundred-millisecond HASTE imaging time (10). Because of this dependence on the cardiac cycle, it is necessary to cardiac-gate the scanning and trigger to the ECG signal.

\section{Data Acquisition}

Imaging experiments were performed on seven healthy human subjects after they provided informed consent. The University of California-San Diego Human Subjects Committee approved the research protocol, and the guidelines proposed by the medical institutional review board were followed. All MRI studies were performed on a Siemens Magnetom Vision 1.5T whole-body system (Siemens Medical Systems, Erlangen, Germany). Each subject was placed in a head-first, supine position into the scanner, after a four-element phased-array body coil was positioned over the thorax. The superior end of the coil was placed directly under the subject's chin to minimize magnetic field dropoff in the apical sections of the lung. The subject was connected to the scanner's ECG hardware, which allowed cardiac gating of the acquisitions and monitoring of the cardiac period (R-R).

To test the basic principles of the technique, we performed multiple ASL experiments with various TIs $(20,21)$ to measure the inflow of tagged blood. These experiments were analogous to previous experiments performed in the brain (22), but involved an experimental design that explicitly accounted for the pulsatility of pulmonary flow. The general organization of the timing is shown in Fig. 1. In these cardiac-gated acquisitions, the scanner triggers off of the upstroke of the ECG Q-wave. The duration between the trigger and the inversion pulse was defined as $T_{e c g}$, and the duration between the inversion pulse and onset of imaging was defined as TI. The timing of the inversion pulse was varied. However, the total time, $T_{t o t},\left(T_{t o t}=T_{e c g}\right.$ + TI), was constant for each subject, so that the image was always collected during diastole of the second cardiac cycle. TI and $T_{\text {ecg }}$ varied through the experiment, but the tag was always applied after the first systolic period (i.e., $T_{\text {ecg }}>T_{\text {sys }}$ ). Choosing $T_{\text {ecg }}$, TI, and $T_{t o t}$ in this manner ensured that at most only one systolic period could deliver tagged spins to the imaging slice (i.e., for long TI the tagging pulse was applied between the two systolic periods, while for short TI the tagging pulse was applied after the second systolic period). The duration of the R-R interval was obtained from the ECG. For the purposes of choosing $T_{\text {tot }}$ and the minimum $T_{e c g}, T_{\text {sys }}$ was approximated as one-third the R-R interval (22). Figure 1 depicts the timing of a typical pulmonary ASL scan, repeated twice, during the same breath-hold (11). A delay of roughly $4 \mathrm{~s}$ was inserted between the two image acquisitions, which allowed longitudinal magnetization to return to steady state. The total scan time using this approach was roughly 8-10 s.

To minimize misregistration between perfusion maps, the subjects were trained to hold their breath at the same point during expiration (functional residual capacity 


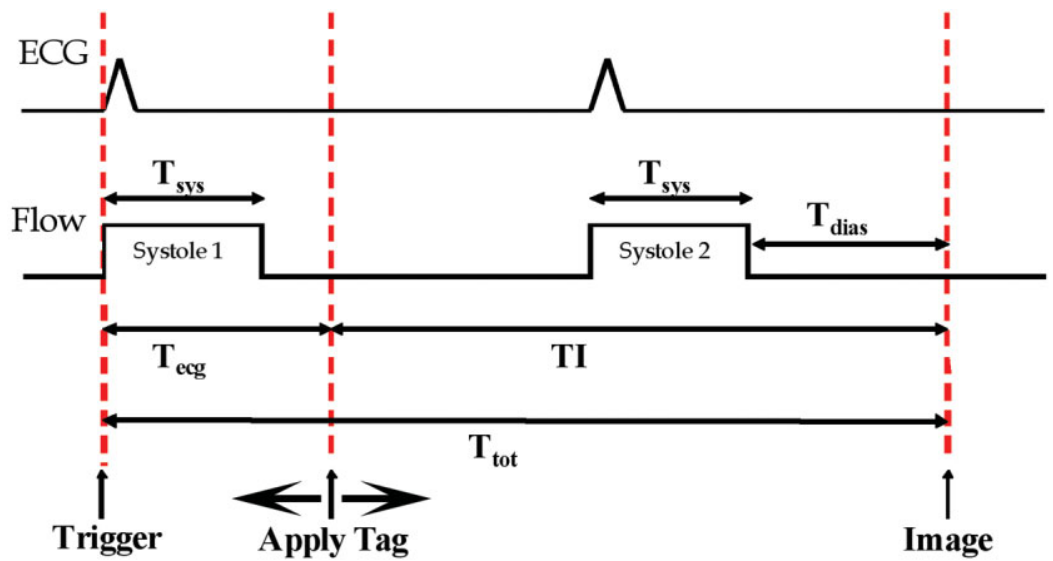

FIG. 1. Timing diagram for pulmonary ASL experiments. MRI hardware triggers off the upstroke of the ECG Q-wave to start scanning. After a delay, $T_{\text {ecg }}$, the tagging pulse is applied, inverting water spins in arterial blood. Another delay, TI, allows delivery of inverted spins to tissue in the imaging plane. Imaging then commences to create either the control or tag image (depending on the nature of the inversion pulse). The timing of the tagging pulse can be varied (horizontal arrows), but the total imaging time is fixed such that imaging always occurs during the second diastolic period. $T_{\text {ecg }}$ must be greater than $T_{\text {sys }}$ to ensure that only one systolic period (systole 2) delivers tagged blood to the imaging plane. The flow profile has been simplified for descriptive purposes.
(FRC)). Multiple, coronal, perfusion-weighted imaging maps were acquired with slices positioned in the posterior lung. Additional imaging parameters were as follows: $\mathrm{TE}=36 \mathrm{~ms}$, FOV $=350-400 \mathrm{~mm}$, slice thickness $=$ $15 \mathrm{~mm}$, and matrix size $=128 \times 256$.

In addition to the ASL scans, a single 2D fast low-angle shot (FLASH) acquisition with the same slice parameters was performed. This scan was used to calculate the global calibration factor, as discussed below. The imaging parameters for the 2D FLASH sequence were as follows: $T_{\text {ecg }}=$ $100, \mathrm{TR}=80-112 \mathrm{~ms}, \mathrm{TE}=3.1-4.8 \mathrm{~ms}$, FOV $=350-$ $400 \mathrm{~mm}$, slice thickness $=15 \mathrm{~mm}$, flip angle $=30^{\circ}$, and matrix size $=128 \times 256$.

\section{Modeling the ASL Experiment}

The primary measurement in an ASL experiment is the subtraction of control and tag images to isolate the signal of delivered blood. To model the ASL signal difference, $\Delta S$, we adapted the general kinetic model (GKM) that was originally developed to analyze the ASL signal in the brain (22). The current analysis explicitly takes into account the high pulsatility of pulmonary flow and the gated acquisition scheme used in the current experiments. In general, our goal was to relate the measured ASL signal in a voxel to the local PBF, $F$, defined as the volume of blood delivered to a volume of tissue per second $(\mathrm{ml} \mathrm{blood} / \mathrm{ml}$ tissue/s) averaged over the cardiac cycle. If the image voxel volume is $V_{\text {voxel }}$, then the volume of blood delivered to a voxel in one cardiac cycle $\left(T_{R R}\right)$ is:

$$
V=F V_{\text {voxel }} T_{R R}
$$

In the pulmonary vasculature the flow is highly pulsatile, and most of the blood is delivered during systole.

For the ASL experiment we assume that 1) the pulses applied in the tag and control parts of the experiment have identical effects on the spins within the image plane, so the difference signal $\Delta S$ (control-tag) arises only from the magnetization difference of blood that was in the tagging band when the inversion pulse was applied, and delivered to the image voxel during the interval TI; and 2) all tagged blood delivered to a voxel remains in the blood compartment of the voxel at the time of measurement (i.e., no exchange with extravascular water and no venous clearance of tagged water molecules).

With these assumptions, $\Delta S$ is proportional to the volume of tagged blood $\left(\mathrm{V}_{\mathrm{ASL}}\right)$ that was delivered from the tagging band to the voxel during the interval TI. If TI = $T_{R R}$, the volume $V_{A S L}$ will approach the true volume of blood delivered during one cardiac cycle. However, $V_{A S L}$ will underestimate the true volume of blood delivered, because of the physical gap between the edge of the imaged slice and the edge of the tagging region. The blood in this gap that will be delivered to a particular image voxel is not tagged, and as such does not contribute to the ASL signal. If we define $V_{\text {gap }}$ as the volume of blood within the gap that will ultimately be delivered to the capillary bed within a voxel, then $V_{A S L}=V-V_{\text {gap }}$. We can also define the effect of the gap in terms of a transit delay, $\delta t$, which is the time required for the tagged blood to cross the gap between the tagging band and the imaged slice. The effect of a transit delay has received considerable attention in ASL applications in the brain $(23,24)$.

We will discuss this systematic error further below, but for now we consider how the signal, $\Delta S$, is calibrated in physiological units. To do this, it is useful to define $\Delta S_{B}$ as the signal difference that would be observed in a voxel completely filled with tagged blood for the timing parameters of the experiment. Then the measured ASL signal can be written as:

$$
\Delta S=\frac{V_{A S L}}{V_{\text {voxel }}} \Delta S_{B}
$$

At time TI, the longitudinal magnetization is tipped into the transverse plane and measured with an acquisition with echo time (TE), so the reference blood signal is:

$$
\Delta S_{B}(T I)=2 M_{0 B} e^{-T I / T_{1}} e^{-T E / T_{2}}
$$

where $M_{\mathrm{O} B}$ is the equilibrium magnetization of blood expressed in imager units, and $T_{1}$ and $T_{2}$ are the longitudinal and transverse relaxation times of blood, respectively, so that $M_{\mathrm{O} B} e^{-T E / T 2}$ is the fully relaxed signal generated by a voxel full of blood after a $90^{\circ}$ pulse. For this study a $T_{1}$ 
value of $1.3 \mathrm{~s}$ and a $T_{2}$ value of $254 \mathrm{~ms}$ were used, based on earlier studies $(13,25)$.

From these relationships, a simple way to estimate $F$ is to perform a single ASL measurement with $\mathrm{TI}=T_{R R}$, use Eqs. [2] and [3] to estimate $V_{A S L}$ from the measured data, and assuming $V_{A S L}$ is approximately equal to $V$, to calculate $F$ from Eq. [1]. This single-subtraction approach will underestimate true $\mathrm{PBF}$ with an error that depends on $V_{\text {gap }}$.

An alternative multiple-subtraction approach, following earlier modeling work on ASL for CBF measurements (22), uses measurements of the curve of $\Delta S$ as a function of TI. To model this curve we must make further assumptions about the time dependence of PBF. We assume highly pulsatile flow, such that during systole the flow has a constant value $F_{\text {sys }}$, and during diastole the flow is zero. Then as TI is varied from low values to high values, there will be an initial duration, $\Delta t$, during which no tagged blood arrives. This is followed by a linear increase of $\Delta S$ while tagged blood is delivered during systole. After a time, $\tau$, no more tagged blood is delivered. Mathematically, the ASL signal can be modeled as:

$$
\begin{aligned}
\Delta S(T I) & =0 & & (T I<\Delta t) \\
& =F_{\text {sys }}(T I-\Delta t) \Delta S_{B}(T I) & & (\Delta t \leq T I \leq \Delta t+\tau) \\
& =F_{\text {sys }} \tau \Delta S_{B}(T I) & & (\Delta t+\tau \leq T I)
\end{aligned}
$$

where the parameter $\Delta t$ is the earliest TI where the signal begins to increase, and $\tau$ is the apparent duration of arrival of tagged spins. This form of the equations is identical to one used previously to model the ASL signal in the brain (22). However, because of the pulsatile nature of pulmonary flow and the gated acquisition, the interpretation of $\Delta t$ and $\tau$ differs from that used in brain studies. In the current experiments the image data were always collected at the same point in the cardiac cycle, and the time of the inversion pulse was varied to change TI. For this reason, the empirical parameter, $\Delta t$, is not the same as the true transit delay across the gap $(\delta t)$, and the apparent duration of the bolus, $\tau$, is not equal to $T_{\text {sys }}$, the true duration of the bolus. Specifically, referring to the timing diagram in Fig. 1, these parameters are:

$$
\begin{gathered}
\Delta t=T_{\text {dias }}+\delta t \\
\tau=T_{\text {sys }}-\delta t
\end{gathered}
$$

With a multiple-subtraction approach, at least two points are measured during the systolic upslope portion of the curve (i.e., when $\Delta t<\mathrm{TI}<\Delta t+\tau$ ), and from these measurements the slope of the curve is calculated to give an estimate of $F_{\text {sys }}$ (24). With the assumption that no flow occurs during diastole, the flow averaged over one cardiac cycle is:

$$
F=F_{s y s} \frac{T_{s y s}}{T_{R R}}
$$

\section{Data Analysis}

Perfusion was measured in the apical, middle, and basal regions of the lung. A total of 20-30 regions of interest
(ROIs) were drawn in each of these sections, and the ROIs were chosen such that signal contribution from large vessels would be minimized. We estimated PBF from the data using both the single- and multiple-subtraction approaches. Equation [4] was fit to the experimental data of $\Delta S$ as a function of TI to determine $\Delta t$ and $\tau$, and to test whether the assumption of pure systolic flow was reasonable (i.e., whether the inflow curve was linear and reached a stable plateau after systolic delivery). The perfusion parameters were fit with a Levenberg-Marquardt leastsquares optimization method, as implemented in MATLAB 6.1 (The Mathworks, Natick, MA, USA). The average flow per cardiac cycle, $F$, was computed from $F_{\text {sys }}$ by approximating $T_{\text {sys }}$ as $\tau$ and applying Eq. [6]. The singlesubtraction analysis was performed using the TI value closest to R-R duration, and applying Eqs. [1]-[3]. In these calculations it was assumed that $V_{A S L}=V$, so the effect of the gap between the image plane and the tagging region was neglected.

To compute absolute flow in both the multiple- and single-subtraction methodologies, it was necessary to estimate the scaling factor $\Delta S_{B}$, the ASL signal for a voxel filled with tagged blood. As described in Eq. [3], this calculation requires $M_{\mathrm{OB}}$. In principle, $M_{\mathrm{OB}}$ can be estimated by directly measuring the blood signal from a HASTE image acquired without spin labeling and correcting for $T_{2}$ decay by multiplying by $e^{T E / T 2}$. However, because the HASTE imaging sequence does not compensate for flow effects, such a method would be inaccurate. Therefore, a single image was first acquired with the same slice prescription as the ASL images using a flow-compensated sequence (2D-FLASH). This low-flip-angle, short-TE segmented sequence provides a proton density-weighted image and was used to calculate the intrinsic ratio of the equilibrium magnetization of arterial blood $\left(M_{\mathrm{OB}}\right)$ to the equilibrium magnetization of a reference organ, the liver $\left(M_{\mathrm{O} L}\right)$. Small ROIs were drawn in homogeneous regions of the descending aorta and liver tissue. The average signal intensity was measured for each subject and corrected for $T_{2}$ decay (with assumed values of $T_{2}=254 \mathrm{~ms}$ for arterial blood; $T_{2}=40 \mathrm{~ms}$ for liver) to obtain an estimate of the intrinsic ratio $\left(M_{\mathrm{OB}} / M_{\mathrm{OL}}\right)$.

To apply Eqs. [2] and [3], $M_{O B}$ must be expressed in scanner units that are scaled identically to those in the ASL images. To accomplish this, a single ( $\mathrm{TR}=\infty$ ) nonASL HASTE image with the same TE as the ASL HASTE sequence was subsequently acquired. The same ROI was drawn in the liver, and signal intensity was measured and corrected for $T_{2}$ decay. $T_{1}$ decay did not have to be considered, since only one single-shot image was acquired; in other words, longitudinal magnetization was already at its maximum. Using this resultant $M_{O L}$ value, along with the intrinsic ratio determined from the 2D-FLASH data, an equilibrium arterial blood magnetization constant, $M_{O B}$, was determined. Unique $M_{O B}$ values were calculated for each experiment because the phased-array body coil creates an inhomogeneous magnetic field in the anteriorposterior direction and distance to the aorta, and therefore the magnetization at the aortic coronal imaging plane will vary between individuals.

The relationship between the multiple- and single-subtraction rPBFs was compared across all lung regions using 


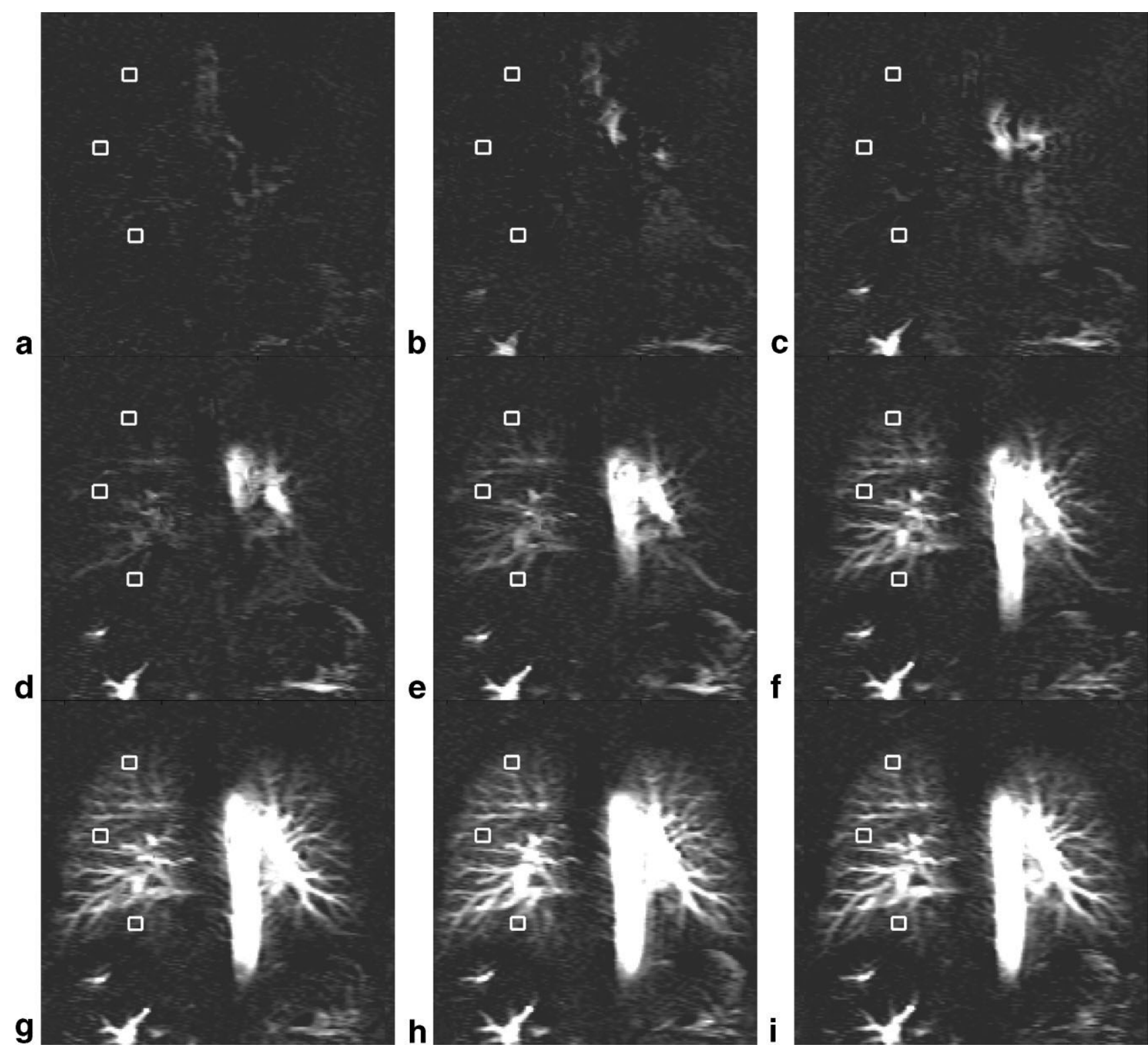

FIG. 2. Pulmonary FAIRER perfusion-weighted images from a healthy volunteer are shown at different Tls: (a) $100 \mathrm{~ms}$, (b) $200 \mathrm{~ms}$, (c) $300 \mathrm{~ms}$, (d) $350 \mathrm{~ms}$, (e) $400 \mathrm{~ms}$, (f) $450 \mathrm{~ms}$, (g) $500 \mathrm{~ms}$, (h) $550 \mathrm{~ms}$, and (i) $600 \mathrm{~ms}$. Evident from this series is the transit delay ( $\Delta t$ ) in a-c. Very few relaxed spins are delivered in the first $300 \mathrm{~ms}$, resulting in minimal lung signal. As TI increases beyond $350 \mathrm{~ms}$, enhancement is clearly visible as relaxed spin delivery increases, since more and more of systole 2 is included. The three white ROls are representative of those used for rPBF calculations in apical, middle, and basal sections of the lung (see Fig. 3 for data points and GKM fit).

linear regression. The effects of intersubject differences on the dependent and independent variables were controlled for by assigning dummy variables to encode the different subjects. A multiple regression analysis (Statview 4.1, SAS Institute Inc., Cary, NC, USA) was then performed (26). Alpha was accepted at $P<0.05$, two-tailed.

\section{RESULTS}

Figure 2 shows representative FAIRER perfusion-weighted image maps from a healthy volunteer (subject 1) at six different TIs (100-600 ms). An apparent transit delay, $\Delta t$, can be clearly identified, as tagged spin delivery to pulmonary voxels starts at about TI $=300 \mathrm{~ms}$. As TI increases, a progressive increase in signal intensity is seen, reflecting an increased delivery of tagged spins. Representative ROIs within the apical, middle, and basal portions of the lung are denoted by white boxes. Figure 3 shows the corresponding, representative $\Delta S$ vs. TI curves for subject 1 , fit with the kinetic model for quantitative ASL perfusion imaging (Eq. [4]). The kinetic curves show a consistent pattern of a delay followed by a sharp rise to a maximum, followed by a gradual decline. Table 1 summarizes the average fit parameters for all seven subjects using the multiple-subtraction analysis and fit with the GKM. In all cases an excellent fit to the model was obtained (mean $\mathrm{R}^{2}=0.90 \pm 0.04$ ). Table 2 compares the average rPBF over the cardiac cycle, measured with both the single- and 


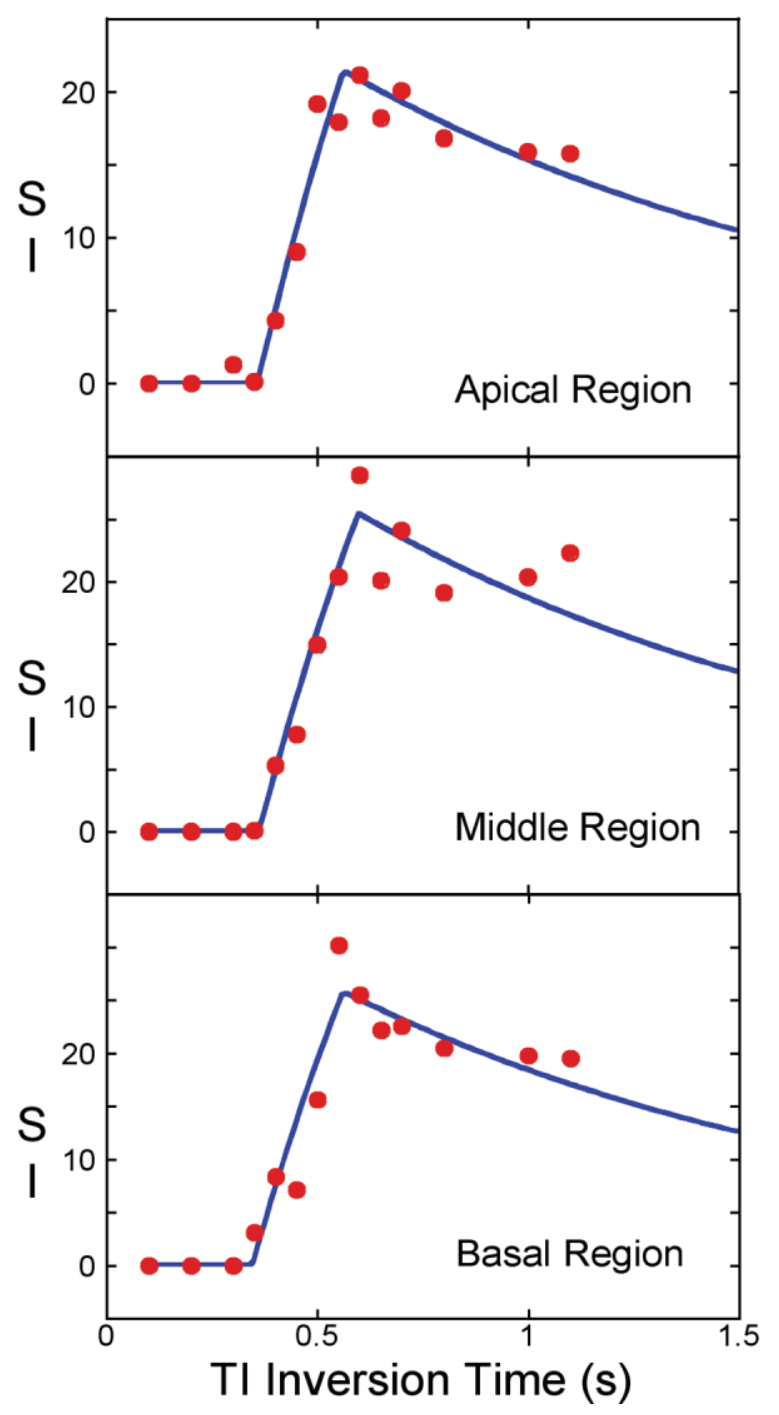

FIG. 3. Application of the GKM for quantitative ASL perfusion imaging to apical, middle, and basal ROI data, from ROls in the healthy human subject shown in Fig. 2. Graphs represent the average pixel intensities in ROls over all TI values (100-1100 ms), fit by GKM (curve). Signal intensity is plotted on the $y$-axis (SI, arbitrary units).

multiple-subtraction analysis techniques. A highly significant relationship was found between flows calculated with the single- and multiple-subtraction analysis techniques $\left(\mathrm{R}^{2}=0.99, P<0.0001\right)$. There was a small but statistically significant difference in $\mathrm{rPBF}$ measured with the two methods. The mean $\mathrm{rPBF}$ averaged $1.70 \pm$ $0.38 \mathrm{ml} / \mathrm{min} / \mathrm{ml}(P<0.05)$ measured with multiple-subtraction analysis, and was approximately $2 \%$ less as measured with the single-subtraction method $(1.66 \pm 0.24$ $\mathrm{ml} / \mathrm{min} / \mathrm{ml} P<0.05$ ).

\section{DISCUSSION}

ASL techniques are now being used in multiple research centers to investigate CBF (27-33); however, applications of ASL techniques to measure PBF are more limited $(14,34,35)$. Although the basic principles are the same when applied to either organ system, there are important differences in the acquisition scheme and data interpretation resulting from the highly pulsatile nature of pulmonary flow and lung motion during respiration. In this study we adapted and analyzed two approaches for quantifying PBF with ASL. The first approach uses a single ASL subtraction of tag and control images, while the second approach uses multiple-subtractions with different TIs. In both the lung and the brain, a critical issue for the accuracy of the technique is the physical gap between the tagging region and the imaged slice. A multiple-subtraction approach was suggested to overcome this problem in the brain by measuring the transit delay caused by the gap. A key result of the current investigation is that this issue is more complicated in the lung, and a multiple-subtraction approach does not fully solve the problem.

In the single-subtraction approach, the ASL signal difference, $\Delta S$, is determined at a single TI with TI $\approx T_{R R}$. This provides an estimate of $V_{A S L}$, the volume of tagged blood delivered to a voxel during one cardiac cycle. This approach makes no assumptions about the flow profile during the cardiac cycle, but it will underestimate true flow due to the spatial gap between the tagging band and the image plane (discussed further below). The multiplesubtraction approach estimates systolic flow from the slope of the measured curve of $\Delta S$ as a function of TI. This analysis requires a more restrictive assumption of constant flow during systole, which is required for a linear inflow curve. Also, to measure flow averaged over the cardiac cycle, an additional assumption must be made to relate systolic flow to average flow. In the analysis presented here, we assumed zero flow during diastole. This assumption was based on the high pulsatility of pulmonary flow (i.e., the majority of flow is concentrated during the systolic period $\left(T_{s y s}\right)$ of the cardiac cycle, with comparatively little inflow during diastole). With this restrictive assumption, an additional measurement of $T_{s y s}$ is required to calculate average flow in one cardiac cycle.

The multiple-subtraction approach adapted here to quantify PBF was based on earlier models developed to quantify CBF (23), and both approaches use the same the mathematical shape to describe the curve of $\Delta S$ vs. TI (Eq. [4]). However, it is important to note that the physical interpretation of this curve differs between the lung and the brain. The gated experimental design we used was required given the highly pulsatile nature of the flow and the restriction of having to collect the data during a breathhold. In typical brain studies the tag and control images are collected over several minutes during different phases of the cardiac cycle. For this reason, pulsatility is rarely considered explicitly in the modeling, with the expectation that the average ASL signal reflects the average flow. In lung experiments we need to account for the pulsatility of blood flow, which introduces an added complexity to the interpretation of the curve of $\Delta S$ vs. TI. Specifically, the apparent transit delay, $\Delta t$, is not the true transit delay, $\delta t$, through the gap between the tagging band and the image plane, and the apparent duration of the tagged bolus, $\tau$ is not the true duration, $T_{\text {sys }}$.

Because of this complexity, the confounding effect of the gap cannot be analyzed in the same way for the lung and brain. In brain studies with FAIR, multiple measurements of the ASL difference signal to characterize the curve $\Delta S$ 
Table 1

Parameter Values from Multiple Subtraction Analysis*

\begin{tabular}{|c|c|c|c|c|c|c|c|c|c|c|c|}
\hline & $T_{R-R}(\mathrm{~s})$ & $T_{\text {tot }}(\mathrm{s})$ & $M_{O B}$ & $\begin{array}{c}f_{\text {sys }} \\
(\mathrm{ml} / \mathrm{min}-\mathrm{ml})\end{array}$ & $\mathrm{SD}(+/-)$ & $\Delta t(\mathrm{~s})$ & $\mathrm{SD}(+/-)$ & $\tau(\mathrm{s})$ & $\mathrm{SD}(+/-)$ & $R^{2}$ & $\mathrm{SD}(+/-)$ \\
\hline \multicolumn{12}{|l|}{ Subject 1} \\
\hline Apical & 0.907 & 1.500 & 1778 & 7.346 & 1.011 & 0.353 & 0.022 & 0.214 & 0.029 & 0.975 & 0.012 \\
\hline Middle & 0.907 & 1.500 & 1778 & 7.212 & 0.681 & 0.348 & 0.024 & 0.230 & 0.028 & 0.948 & 0.028 \\
\hline Basal & 0.907 & 1.500 & 1778 & 6.100 & 0.903 & 0.354 & 0.022 & 0.227 & 0.028 & 0.905 & 0.037 \\
\hline \multicolumn{12}{|l|}{ Subject 2} \\
\hline Apical & 0.900 & 1.500 & 1376 & 4.517 & 0.771 & 0.277 & 0.013 & 0.227 & 0.019 & 0.937 & 0.016 \\
\hline Middle & 0.900 & 1.500 & 1376 & 4.962 & 0.526 & 0.262 & 0.025 & 0.242 & 0.032 & 0.874 & 0.035 \\
\hline Basal & 0.900 & 1.500 & 1376 & 7.592 & 1.105 & 0.287 & 0.007 & 0.235 & 0.036 & 0.905 & 0.050 \\
\hline \multicolumn{12}{|l|}{ Subject 3} \\
\hline Apical & 0.789 & 1.300 & 1945 & 6.048 & 1.075 & 0.280 & 0.019 & 0.197 & 0.040 & 0.963 & 0.021 \\
\hline Middle & 0.789 & 1.300 & 1945 & 6.172 & 0.953 & 0.262 & 0.028 & 0.223 & 0.043 & 0.916 & 0.043 \\
\hline Basal & 0.789 & 1.300 & 1945 & 5.116 & 0.748 & 0.262 & 0.022 & 0.231 & 0.033 & 0.881 & 0.024 \\
\hline \multicolumn{12}{|l|}{ Subject 4} \\
\hline Apical & 0.880 & 1.400 & 1833 & 5.764 & 1.030 & 0.271 & 0.035 & 0.206 & 0.039 & 0.901 & 0.035 \\
\hline Middle & 0.880 & 1.400 & 1833 & 5.743 & 1.289 & 0.276 & 0.026 & 0.213 & 0.045 & 0.887 & 0.035 \\
\hline Basal & 0.880 & 1.400 & 1833 & 6.257 & 1.578 & 0.272 & 0.028 & 0.256 & 0.037 & 0.904 & 0.049 \\
\hline \multicolumn{12}{|l|}{ Subject 5} \\
\hline Apical & 0.936 & 1.500 & 2603 & 6.723 & 0.661 & 0.279 & 0.023 & 0.190 & 0.028 & 0.845 & 0.023 \\
\hline Middle & 0.936 & 1.500 & 2603 & 6.622 & 0.801 & 0.308 & 0.023 & 0.192 & 0.040 & 0.880 & 0.043 \\
\hline Basal & 0.936 & 1.500 & 2603 & 5.792 & 1.164 & 0.301 & 0.025 & 0.270 & 0.031 & 0.859 & 0.032 \\
\hline \multicolumn{12}{|l|}{ Subject 6} \\
\hline Apical & 1.105 & 1.900 & 1729 & 6.864 & 0.740 & 0.327 & 0.048 & 0.404 & 0.062 & 0.912 & 0.047 \\
\hline Middle & 1.105 & 1.900 & 1729 & 7.652 & 0.715 & 0.367 & 0.030 & 0.367 & 0.030 & 0.873 & 0.049 \\
\hline Basal & 1.105 & 1.900 & 1729 & 5.810 & 0.866 & 0.388 & 0.031 & 0.334 & 0.039 & 0.875 & 0.028 \\
\hline \multicolumn{12}{|l|}{ Subject 7} \\
\hline Apical & 0.928 & 1.500 & 1729 & 6.498 & 0.867 & 0.258 & 0.031 & 0.223 & 0.047 & 0.887 & 0.020 \\
\hline Middle & 0.928 & 1.500 & 1729 & 7.332 & 0.945 & 0.263 & 0.028 & 0.259 & 0.058 & 0.855 & 0.038 \\
\hline Basal & 0.928 & 1.500 & 1729 & 7.154 & 1.092 & 0.214 & 0.042 & 0.275 & 0.060 & 0.821 & 0.021 \\
\hline
\end{tabular}

${ }^{*}$ Resultant parameter values after data from multiple-subtraction analysis is fit with the general kinetic model for quantitative ASL perfusion imaging. The $R^{2}$ statistic provides a goodness-of-fit measure. Data points from at least 20 different ROls, in each of the three pulmonary regions (apical, middle, and basal), were averaged and reported. The standard deviation (SD) across the ROls is also reported.

vs. TI provide a direct estimate of $\delta t$, so the transit delays can be taken into account. For gated studies in the lung, $\Delta t$ depends on both $\delta t$ and $T_{\text {dias }}$, the time between the end of the previous systolic period and data acquisition. Furthermore, for these gated acquisitions it is easiest to think about the physical source of the problem in terms of the volume of blood within the gap, rather than as a transit delay problem. Ideally, by choosing $\mathrm{TI}=T_{R R}$, we are measuring the total amount of blood delivered to a voxel during one cardiac cycle. The error comes in because we have not tagged all of the blood that will be delivered during one cardiac cycle. The missing blood is the blood in the gap that was not tagged, or simply, $V_{\text {gap }}$. For this reason the problem is essentially one of anatomy, rather than transit times. If the vascular volume remained unchanged but flow velocity increased, the untagged volume $\left(V_{\text {gap }}\right)$ would remain the same but the transit delay $(\delta t)$ would decrease.

To summarize, the advantages and limitations of the two approaches can be characterized as follows: With the single-subtraction approach using $\mathrm{TI} \approx T_{R R}$, the experiment can be performed quickly, and the volume, $V_{A S L}$, of tagged blood delivered during one cardiac cycle can be measured directly. No assumptions are required about the flow dynamics during the cardiac cycle, which is a powerful feature given the complex flow patterns in the lung. However, the gap transit delay will cause the $V_{A S L}$ measurement to underestimate true PBF, with the fractional underestimation as:

$$
\text { Fractional error }=\frac{V_{G A P}}{V_{G A P}+V_{A S L}}
$$

It follows that as long as $V_{\text {gap }}$ remains small compared to $V_{A S L}$, the error introduced by the gap will also be small. Further experiments are required to estimate the magnitude of this error in practical applications.

The multiple-subtraction approach, in principle, provides a measurement of $F_{\text {sys }}$, the flow during systole. Measurement of $F_{\text {sys }}$ is independent of the physical gap between the imaging slice and tagging region, since the calculation depends only on the slope of the measured curve of $\Delta S$ vs. TI after tagged blood begins to arrive in the voxel. Since several TI data points are needed to characterize the upslope portion of the curve, the multiple-subtraction approach requires a more time-consuming experiment than the single-subtraction approach. Also, the multiple-subtraction approach requires additional assumptions about the flow dynamics during the cardiac cycle. In the analysis described here, we assumed the extreme case of constant flow during systole and zero flow during diastole. This produces a linear inflow curve of $\Delta S$ as a function of TI, and the time-averaged flow is then related to systolic flow 
Table 2

Multiple-Subtraction and Single-Subtraction $\mathrm{rPBF}^{\star}$

\begin{tabular}{ccccc}
\hline & $\begin{array}{c}f_{M S} \\
(\mathrm{ml} / \mathrm{min}-\mathrm{ml})\end{array}$ & SD $(+/-)$ & $\begin{array}{c}f_{s s} \\
(\mathrm{ml} / \mathrm{min}-\mathrm{ml})\end{array}$ & $\mathrm{SD}(+/-)$ \\
\hline Subject 1 & & & & \\
Apical & 1.731 & 0.238 & 1.860 & 0.393 \\
Middle & 1.832 & 0.173 & 1.872 & 0.327 \\
$\begin{array}{c}\text { Basal } \\
\text { Subject 2 }\end{array}$ & 1.528 & 0.226 & 1.341 & 0.292 \\
Apical & 1.140 & 0.194 & 0.963 & 0.173 \\
Middle & 1.332 & 0.141 & 1.211 & 0.280 \\
Basal & 1.983 & 0.289 & 1.950 & 0.309 \\
Subject 3 & & & & \\
Apical & 1.509 & 0.268 & 1.581 & 0.284 \\
Middle & 1.744 & 0.269 & 1.813 & 0.292 \\
Basal & 1.496 & 0.219 & 1.397 & 0.162 \\
Subject 4 & & & & \\
Apical & 1.352 & 0.241 & 1.231 & 0.189 \\
Middle & 1.392 & 0.312 & 1.272 & 0.227 \\
Basal & 1.817 & 0.458 & 1.835 & 0.471 \\
Subject 5 & & & & \\
Apical & 1.368 & 0.134 & 1.312 & 0.258 \\
Middle & 1.361 & 0.165 & 1.224 & 0.376 \\
Basal & 1.669 & 0.336 & 1.575 & 0.372 \\
Subject 6 & & & & \\
Apical & 2.513 & 0.271 & 2.572 & 0.424 \\
Middle & 2.544 & 0.238 & 2.510 & 0.389 \\
Basal & 1.756 & 0.262 & 1.708 & 0.226 \\
Subject 7 & & & & \\
Apical & 1.564 & 0.209 & 1.571 & 0.372 \\
Middle & 2.048 & 0.264 & 2.076 & 0.532 \\
Basal & 2.123 & 0.324 & 1.934 & 0.557 \\
\hline
\end{tabular}

${ }^{*}$ Comparison of average rPBF per cardiac cycle calculated from multiple-subtraction $\left(f_{M S}\right)$ and single-subtraction $\left(f_{S S}\right)$ analysis methods, across multiple ROls (between 20 and 30) in apical, middle, and basal regions. The standard deviation (SD) across the ROls is also reported.

in a simple way (Eq. [6]). The true pulmonary profile will undoubtedly deviate from this square-wave profile, especially with pathology, and thus introduce error into the $F_{\text {sys }}$ calculation. Furthermore, in order to convert the measurement of $F_{\text {sys }}$ into an estimate of $F$, the average flow over the cardiac cycle, an additional precise measurement of systolic duration $T_{\text {sys }}$ is required. Even with the assumption of an idealized rectangular-wave profile, $T_{\text {sys }}$ will not be equal to $\tau$, as discussed in the Materials and Methods section, due to the physical gap. While the approximation of $T_{s y s}$ as one-third $T_{R-R}$ is suitable for the purposes of choosing $T_{\text {tot }}$, it is not exact enough to accurately calculate flow. Consequently, if $F$ is calculated by estimating $T_{\text {sys }}$ as $\tau$, this will result in the same fractional underestimation as the single-subtraction approach (Eq. [6]). To avoid this problem, one could obtain a more precise measurement of $T_{\text {sys }}$ by alternative MRI techniques; however, such approaches would further complicate the experiment and introduce additional scans. In short, even a multiple-subtraction approach will become sensitive to gap transit delay errors in calculating the average flow $F$. As a result, one of the primary advantages of a multiplesubtraction approach over a single-subtraction approachinsensitivity to gap transit delay errors-is lost if $T_{\text {sys }}$ is approximated as $\tau$.
In addition to the assumptions that are specific to the two approaches, the methods share two assumptions in common: 1) that the exchange of tagged water between vasculature and tissue is negligible during TI, and 2) that entering tagged water stays in the vascular tree and does not clear through the venous circulation during TI. With these assumptions, the measured ASL difference signal is proportional to the volume of delivered tagged blood, $V_{A S L}$, but to no other local parameters. In this sense, $\Delta S$ can be interpreted as a measure of PBF that lacks only a global calibration factor $\left(\Delta S_{B}\right)$ to convert the signal measurement into absolute flow units. The global scaling factor depends only on properties of blood, and can be estimated.

If tagged water spins in blood do exchange with tissue water spins, $T_{1}$ decay must be modeled with two rate constants: the $T_{1}$ of arterial blood $\left(T_{1 b}\right)$ and the $T_{1}$ of lung tissue $\left(T_{1}\right)$. The longer the TI delay, the more spins will exchange with tissue, eventually approaching equilibrium. The assumption made in these studies, however, is that even at the longest TIs very little blood water will exchange with the tissue. Because the $T_{1}$ of tissue is typically shorter than that of blood, perfusion will be slightly underestimated if exchange occurs.

In the simplest terms, perfusion calculation using the ASL technique relies on knowing the volume of tagged spins delivered to the imaging slice, and the duration of time needed to deliver that particular volume. Voxel signal intensity will be directly proportional to volume of spins delivered, as long as the delivered spins do not leave the voxel during the delivery time (TI). Previous theoretical work involving the measurement of CBF suggested that this phenomenon is the least important systematic error in ASL imaging and has a virtually negligible effect (24). Blood velocities in the lung, however, are typically faster than those in the brain $(15,24)$, and voxels containing large vessels with fast-moving blood might have spins delivered and subsequently cleared during TI. Therefore, we made an effort to choose ROIs in low-flow regions of the lung and hence avoid large vessels. The peripheral areas of the lung were usually free of large vessels.

A notable feature of the ASL difference images is that many large vessels appear bright. Strictly speaking, this signal does not represent quantitative PBF (i.e., the delivery of blood to the capillary bed of the lung). Tagged blood in a large vessel within a voxel cannot be properly counted as perfusion within that voxel if it is being delivered to capillary beds in more distal regions. In fact, if a voxel contains a segment of a large vessel that is filled with tagged blood, the ASL signal will reflect blood volume rather than blood flow. In the case of smaller vessels, in which none of the tagged blood has cleared, the ASL signal reflects perfusion in the same way that microspheres reflect perfusion. For these reasons, the bright vessels could be considered to be an artifact of the perfusion image. One way to deal with this problem is to apply bipolar gradient pulses to destroy the signal of fast-flowing blood. Such "flow weighting" can be effective in removing signal from the largest vessels. This approach, however, aggravates the problem of the transit delay across the gap. Effectively, the transit delay will be longer because tagged spins must not only cross the gap and enter the voxel, but also move along 
the vascular tree to vessels with slower flow before they generate a measurable signal. Effectively, the volume of missing blood $V_{\text {gap }}$ will grow because some of the blood that was originally tagged will remain in the faster-flowing vessels of the voxel at imaging time, but will not generate an ASL signal. Thus there is a trade-off involved in trying to remove the large vessel signal while maintaining adequate tissue-perfusion signal. The best quantitative approach remains to be determined. In addition, it may be that the large vessel signal carries useful diagnostic information, even if it interferes with a strict interpretation of the signal as pulmonary perfusion.

The results of this study show that flows calculated with the single- and multiple-subtraction approaches yield comparable flow values, and these values are in good agreement with other published measurements. In the simplest terms, the mean pulmonary perfusion can be estimated as cardiac output divided by the weight of the human lung, since in healthy individuals the lungs receive the entire cardiac output. The normal resting value of cardiac output is about $5 \mathrm{l} / \mathrm{min}$ (36), and the weight of the human lung is about $1 \mathrm{~kg}$ (37); therefore, the perfusion of the lung is $5 \mathrm{ml} / \mathrm{g} / \mathrm{min}$. Measurements of regional pulmonary perfusion obtained with a variety of techniques also approximate this value. For example, using ${ }^{1} \mathrm{H}$ spin labeling MRI, Wang et al. (14) observed perfusion rates in healthy lungs of 4-6 $\mathrm{ml} / \mathrm{min} / \mathrm{g}$. However, many measures of PBF, including the present one, express perfusion per volume of lung tissue and therefore can only be compared when the lung volume at which the imaging takes place is known and a value for lung density at that lung volume is assumed. Since the density of the right human lung at FRC is about $0.3 \mathrm{~g} / \mathrm{ml}$ (37), this would provide a comparable value of $5.5 \mathrm{ml} / \mathrm{min} / \mathrm{g}$ for pulmonary perfusion for our average single-subtraction measurement of $1.66 \mathrm{ml} / \mathrm{min} /$ ml. Data obtained using PET scanning (in which data are acquired during quiet breathing, resulting in slightly lower lung density) indicate a regional pulmonary perfusion of $1.9 \mathrm{ml} / \mathrm{min} / \mathrm{ml}$ (38), providing a comparable value of $6.3 \mathrm{ml} / \mathrm{min} / \mathrm{g}$.

Based on the above discussion, both single- and multiple-subtraction methods are susceptible to transit delay errors, and the multiple-subtraction approach is also susceptible to error caused by a non-ideal flow profile. Therefore, without additional experiments, it is difficult to comment on the accuracy of these reported values. Furthermore, because we approximated $T_{\text {sys }}$ as $\tau$ when we computed $f$ with the multiple-subtraction approach, the fractional error caused by the physical gap will be given by Eq. [6], just as with the single-subtraction approach. Therefore, differences between average flows calculated with the single- and multiple-subtraction methods are likely due primarily to error caused by a non-ideal flow profile. The fact that the results from both methods are highly correlated, with only a $2 \%$ difference in the calculated flow values, suggests that an idealized flow-profile assumption is reasonable, at least in normal physiology. However, this may change in pathology, which would make the profileinsensitive single-subtraction approach the method of choice.

In conclusion, we have presented the quantitative results of MR perfusion experiments in normal subjects us- ing an ASL-FAIRER imaging scheme. Single- and multiple-subtraction techniques were theoretically and experimentally explored. Pulmonary ASL approaches are markedly different from ASL approaches in the brain, in terms of both data acquisition and analysis, primarily because of the high pulsatility and strong cardiac-cycle dependence of PBF. In the brain, a multiple-subtraction approach adequately addresses errors caused by physical gaps between tagging and imaging volumes, thus facilitating perfusion quantification. In the lung, however, rather than simplifying perfusion quantification, a multiple-subtraction approach introduces a restrictive assumption regarding flow delivery. Furthermore, such an approach in the lung requires an additional accurate $T_{\text {sys }}$ measurement, which is also complicated by the gap transit delay. While a single-subtraction approach does not address the physical gap, which leads to an underestimation in perfusion, it does not depend on the shape of the bolus inflow profile, and can provide a flow measurement with a single tag-pair acquisition. As long as $V_{\text {gap }}$ is relatively small compared to one cardiac cycle's worth of delivered blood, a physiologically useful value for average pulmonary flow can be estimated. More experimental work is needed to address the practical significance of this question.

ASL has great potential for evaluating $\mathrm{rPBF}$, which can be useful for diagnosing disease, monitoring disease progression, and evaluating disease response to therapy. Additional characterization of the pulmonary perfusion response is necessary to determine the magnitude of perfusion error and further develop an optimal quantitative approach that will be feasible for both research and clinical settings.

\section{REFERENCES}

1. Detre JA, Leigh JS, Williams DS, Koretsky AP. Perfusion imaging. Magn Reson Med 1992;23:37-45.

2. Williams DS, Detre JA, Leigh JS, Koretsky AP. Magnetic resonance imaging of perfusion using spin inversion of arterial water. Proc Nat Acad Sci USA 1992;89:212-216.

3. Edelman RR, Siewert B, Darby DG, Thangaraj V, Nobre AC, Mesulam MM, Warach S. Qualitative mapping of cerebral blood flow and functional localization with echo-planar MR imaging and signal targeting with alternating radio frequency. Radiology 1994;192:513-520.

4. Kim SG. Quantification of relative cerebral blood flow change by flowsensitive alternating inversion recovery (FAIR) technique: application to functional mapping. Magn Reson Med 1995;34:293-301.

5. Alsop DC, Detre JA. Reduced transit-time sensitivity in noninvasive magnetic resonance imaging of human cerebral blood flow. J Cereb Blood Flow Metab 1996;16:1236-1249.

6. Wong EC, Buxton RB, Frank LR. Quantitative perfusion imaging using EPISTAR and FAIR. In: Proceedings of the 4th Annual Meeting of ISMRM, New York, NY, USA, 1996. p 13.

7. Frank LR, Wong EC, Haseler LJ, Buxton RB. Dynamic imaging of perfusion in human skeletal muscle during exercise with arterial spin labeling. Magn Reson Med 1999;42:258-267.

8. Poncelet BP, Koelling TM, Schmidt CJ, Kwong KK, Reese TG, Ledden P, Kantor HL, Brady TJ, Weisskoff RM. Measurement of human myocardial perfusion by double-gated flow alternating inversion recovery EPI. Magn Reson Med 1999;41:510-519.

9. Roberts DA, Detre JA, Bolinger L, Insko EK, Lenkinski RE, Pentecost MJ, Leigh Jr JS. Renal perfusion in humans: MR imaging with spin tagging of arterial water. Radiology 1995;196:281-286.

10. Mai VM, Berr SS. MR perfusion imaging of pulmonary parenchyma using pulsed arterial spin labeling techniques: FAIRER and FAIR. J Magn Reson Imaging 1999;9:483-487. 
11. Mai VM, Hagspiel KD, Christopher JM, Do HM, Altes T, Knight-Scott J, Stith AL, Maier T, Berr SS. Perfusion imaging of the human lung using flow-sensitive alternating inversion recovery with an extra radiofrequency pulse (FAIRER). Magn Reson Imaging 1999;17:355-361.

12. Edelman RR, Hatabu H, Tadamura E, Kim D, Prasad PV, Buxton RB. Pulmonary perfusion: nonivasive imaging and quantification by magnetic resonance. In: Proceedings of the 4th Annual Meeting of ISMRM, New York, NY, USA, 1996. p 1314.

13. Hatabu H, Tadamura E, Prasad PV, Chen Q, Buxton R, Edelman RR Noninvasive pulmonary perfusion imaging by STAR-HASTE sequence. Magn Reson Med 2000;44:808-812.

14. Wang T, Schultz G, Hebestreit H, Hebestreit A, Hahn D, Jakob PM. Quantitative perfusion mapping of the human lung using $1 \mathrm{H}$ spin labeling. J Magn Reson Imaging 2003;18:260-265.

15. Chen Q, Siewert B, Bly BM, Warach S, Edelman RR. STAR-HASTE: perfusion imaging without magnetic susceptibility artifact. Magn Reson Med 1997;38:404-408.

16. Harris P, Heath D. The human pulmonary circulation. Edinburgh: Churchill Livingston; 1986.

17. Haacke EM, Brown RW, Thompson MR, Venkatesan R. Magnetic resonance imaging: physical principles and sequence design. New York: John Wiley and Sons; 1999. p 118.

18. Keifer B, Grassner J, Hausmann R. Image acquisition in a second with a half-Fourier acquisition single-shot turbo spin echo. In: Proceedings of the 2nd Annual Meeting of ISMRM, San Francisco, CA, USA, 1994.

19. Mai VM, Chen Q, Bankier AA, Zhang M, Hagspiel KD, Berr SS, Edelman RR. Imaging pulmonary blood flow and perfusion using phasesensitive selective inversion recovery. Magn Reson Med 2000;43:793795.

20. Bolar DS, Levin DL, Hopkins SR, Frank LR, Liu TT, Buxton RB, Wong EC. A single-subtraction method for quantitative ASL pulmonary perfusion analysis. In: Proceedings of the 10th Annual Meeting of ISMRM, Honolulu, HI, USA, 2002.

21. Bolar DS, Levin DL, Hopkins SR, Frank LR, Mai VM, Chen Q, Buxton RB. Application of a general kinetic model to ASL-FAIRER quantitative MR pulmonary perfusion imaging. In: Proceedings of the Society of Thoracic Radiology, San Francisco, CA, USA, 2002.

22. Cabin HS. The heart and circulation. In: Zaret BL, Moser M, Cohen LS, editors. Yale University School of Medicine heart book. New York: Hearst Books; 1992. p 5.

23. Wong EC, Buxton RB, Frank LR. Quantitative imaging of perfusion using a single subtraction (QUIPSS and QUIPSS II). Magn Reson Med 1998;39:702-708.

24. Buxton RB, Frank LR, Wong EC, Siewert B, Warach S, Edelman RR. A general kinetic model for quantitative perfusion imaging with arterial spin labeling. Magn Reson Med 1998;40:383-396.
25. Barth M, Moser E. Proton NMR relaxation times of human blood samples at $1.5 \mathrm{~T}$ and implications for functional MRI. Cell Mol Biol (Noisyle-Grand) 1997;43:783-791.

26. Glantz SA, Slinker BK. Primer of applied regression and analysis of variance. New York: McGraw Hill; 1990.

27. Golay X, Petersen ET, Hui F. Pulsed star labeling of arterial regions (PULSAR): a robust regional perfusion technique for high field imaging. Magn Reson Med 2005;53:15-21.

28. Mildner T, Zysset S, Trampel R, Driesel W, Moller HE. Towards quantification of blood-flow changes during cognitive task activation using perfusion-based fMRI. Neuroimage 2005;27:919-926.

29. Hoge RD, Franceschini MA, Covolan RJ, Huppert T, Mandeville JB Boas DA. Simultaneous recording of task-induced changes in blood oxygenation, volume, and flow using diffuse optical imaging and arterial spin-labeling MRI. Neuroimage 2005;25:701-707.

30. Jahng GH, Song E, Zhu XP, Matson GB, Weiner MW, Schuff N. Human brain: reliability and reproducibility of pulsed arterial spin-labeling perfusion MR imaging. Radiology 2005;234:909-916.

31. Garraux G, Hallett M, Talagala SL. CASL fMRI of subcortico-cortical perfusion changes during memory-guided finger sequences. Neuroimage 2005;25:122-132.

32. Wang J, Zhang Y, Wolf RL, Roc AC, Alsop DC, Detre JA. Amplitudemodulated continuous arterial spin-labeling 3.0-T perfusion MR imaging with a single coil: feasibility study. Radiology 2005;235:218-228.

33. Stefanovic B, Warnking JM, Kobayashi E, Bagshaw AP, Hawco C, Dubeau F, Gotman J, Pike GB. Hemodynamic and metabolic responses to activation, deactivation and epileptic discharges. Neuroimage 2005; 28:205-215.

34. Hopkins SR, Garg J, Bolar DS, Balouch J, Levin DL. Pulmonary blood flow heterogeneity during hypoxia and high-altitude pulmonary edema. Am J Respir Crit Care Med 2005;171:83-87.

35. Lin YR, Wu MT, Huang TY, Tsai SY, Chung HW, Mai VM, Chen CY, Pan HB. Comparison of arterial spin labeling and first-pass dynamic contrast-enhanced MR imaging in the assessment of pulmonary perfusion in humans: the inflow spin-tracer saturation effect. Magn Reson Med 2004;52:1291-1301.

36. West J. Respiratory physiology—the essentials. 5th ed. Baltimore: Williams \& Wilkins; 1995. p 39-43.

37. Brudin LH, Rhodes CG, Valind SO, Wollmer P, Hughes JM. Regional lung density and blood volume in nonsmoking and smoking subjects measured by PET. J Appl Physiol 1987;63:1324-1334.

38. Brudin LH, Rhodes CG, Valind SO, Jones T, Hughes JM. Interrelationships between regional blood flow, blood volume, and ventilation in supine humans. J Appl Physiol 1994;76:1205-1210. 